\title{
ORIGINAL RESEARCH \\ Cerebral Microbleeds on MR Imaging: Comparison between 1.5 and $7 T$
}

\section{M.M.A. Conijn \\ M.I. Geerlings \\ G.-J. Biessels \\ T. Takahara \\ T.D. Witkamp}

J.J.M. Zwanenburg

P.R. Luijten

J. Hendrikse
BACKGROUND AND PURPOSE: The detection of microbleeds differs strongly between studies, due to differences in scan protocol. This study aims to compare the visualization of microbleeds with $3 D$ T2* ${ }^{*}$ weighted imaging at $1.5 \mathrm{~T}$ with $3 \mathrm{D}$ dual-echo $\mathrm{T} 2{ }^{*}$-weighted imaging at $7 \mathrm{~T}$.

MATERIALS AND METHODS: Thirty-four patients (29 male; mean age, $58 \pm 12$ years) with atherosclerotic disease from the Second Manifestations of ARTerial Disease study were included. 3D T2* weighted imaging at $1.5 \mathrm{~T}$ and dual-echo $\mathrm{T} 2{ }^{*}$-weighted imaging at $7 \mathrm{~T}$ were done in all patients. The presence and number of definite microbleeds were recorded on minimal intensity projections. Interand intraobserver reliability was assessed with Cohen $\kappa$ test and the ICC. The difference in presence and number of microbleeds was tested with the McNemar test and Wilcoxon signed rank test.

RESULTS: The interobserver ICC at 7T was 0.61 and the intraobserver ICC was 0.94 , whereas at 1.5T the interobserver ICC was 0.50 and the intraobserver ICC was 0.59 . Microbleeds were detected in significantly more patients on $7 \mathrm{~T}(50 \%)$ than on $1.5 \mathrm{~T}$ scans $(21 \%)(P=.001)$. The number of microbleeds was also higher at 7T (median, 0.5; range, $0-5$ ) than on 1.5T (median, 0.0; range, 0-6) $(P=.002)$.

CoNCLUSIONS: 3D dual-echo T2*-weighted imaging at $7 \mathrm{~T}$ results in better and more reliable detection of microbleeds compared with $3 \mathrm{D} T 2^{*}$-weighted imaging at $1.5 \mathrm{~T}$.

ABBREVIATIONS: GRE = gradient recalled-echo; ICC = intraclass correlation coefficient; MARS = Microbleed Anatomical Rating Scale; MIP = minimum intensity projection; PRESTO = principles of echo shifting with a train of observations; SMART = Second Manifestations of ARTerial disease; SNR = signal-to-noise ratio; SWI = susceptibility-weighted imaging; TE1 = first echo; TE2 = second echo
R ecent studies show that cerebral microbleeds are commonly detected on MR imaging in the general population as well as in specific patient populations, such as patients with cerebral small vessel disease, Moyamoya disease, and other neurologic pathologies. ${ }^{1-8}$ Simultaneously, the evidence about the clinical relevance of microbleeds increases. Cerebral microbleeds seem to be direct markers of vascular disease, because they are associated with hypertensive vasculopathy and cerebral amyloid angiopathy and also with white matter lesions and lacunar infarcts. ${ }^{1,8-10}$ Although still under debate, the presence of microbleeds could be of importance in patients with ischemic stroke receiving anticoagulation, because it might indicate a higher risk of future intracerebral hemorrhage. ${ }^{11-15}$ Furthermore, some studies have shown that microbleeds are associated with cognitive impairment, functional dependence, and death. ${ }^{10,16,17}$

The number of microbleeds detected differs strongly between studies, even between studies with similar study populations. In population-based studies, eg, prevalences range from $5 \%$ to $23 \% .^{3,7}$ Differences in lesion prevalence are probably not only related to the characteristics of the different study populations but also to differences in the MR protocol,

Received September 20, 2010; accepted after revision October 22.

From the Department of Radiology (M.M.A.C., T.T., T.D.W., J.J.M.Z., P.R.L., J.H.), Julius Center for Health Sciences and Primary Care (M.M.A.C., M.I.G.); Department of Neurology (G.-J.B.), Rudolf Magnus Institute of Neuroscience; and Image Sciences Institute (J.J.M.Z., P.R.L.), University Medical Center Utrecht, Utrecht, the Netherlands.

Please address correspondence to M.M.A. Conijn, MD, Department of Radiology, University Medical Center Utrecht, PO Box 85500, 3508 GA Utrecht, the Netherlands; e-mail: m.conijn@umcutrecht.nl

DOI 10.3174/ajnr.A2450 such as differences in scan technique, TR, TE, flip angle, band width, section thickness, and spatial resolution. ${ }^{18,19}$

Microbleeds are paramagnetic, which induces a susceptibility effect on the MR imaging scan. This leads to a fast decay of local T2*-weighted MR imaging signal intensity because of a local inhomogeneity of the field induced by the microbleeds. This makes $\mathrm{T} 2{ }^{\star}$-weighted GRE very sensitive to microbleeds. ${ }^{9,10,20}$ One of the factors in the imaging protocol that influences the visualization of microbleeds is the TE. A longer TE gives more time for dephasing, which enhances the susceptibility effect. This so-called blooming effect causes the microbleeds to appear as hypointense spots that are larger than their actual size. It has been shown that prolonging the TE leads to an increase in diameter of these lesions and also to the detection of an increased number of microbleeds. ${ }^{21}$ However, microbleeds also can be obscured by overlapping structures with a high susceptibility effect, such as veins (deoxyhemoglobin) or the basal ganglia (ferritin deposition). With a longer $\mathrm{TE}$, these structures also increase in size, making it harder to distinguish the microbleeds. Therefore, both a short TE and a long TE may have benefits for the visualization of microbleeds. Besides the TE, the spatial resolution also is important for the visualization of microbleeds. With a higher resolution, partial volume effects are minimized and smaller microbleeds can be visualized. ${ }^{22}$ Another factor that influences the visualization of microbleeds is magnetic field strength. So far, most studies investigating microbleeds used a $1.5 \mathrm{~T}$ MR imaging scanner. However, studies comparing $1.5 \mathrm{~T}$ and $3 \mathrm{~T}$ show a higher number of microbleeds at 3T. ${ }^{18,23}$ As the susceptibility effect scales with the magnetic field, scanning at higher field strengths improves the sensitivity to microbleeds. At ultrahigh field 
strengths, such as 7T, the susceptibility effect further increases as does the SNR. This increase in SNR can be used for higher spatial resolution with increased conspicuity of small hemosiderin deposits that may be obscured by partial volume effects at lower field strengths operating at lower spatial resolution. ${ }^{24}$ Furthermore, scanning at ultrahigh field strengths enables the use of dual-echo $\mathrm{T} 2{ }^{*}$-weighted imaging for the visualization of microbleeds, whereas at lower field strengths this is probably not feasible because the susceptibility effect may not be large enough to visualize the microbleeds at the short TEs. The use of a dual-echo sequence at $7 \mathrm{~T}$ has been shown to combine the benefits of a short TE and a long TE for the visualization of microbleeds in one sequence. ${ }^{25}$ It has not been established whether the use of a dual-echo sequence at $7 \mathrm{~T}$ increases the number of detected microbleeds compared with the commonly used single-echo $\mathrm{T} 2^{\star}$-weighted imaging at lower field strengths. The purpose of our study was to compare the visualization of microbleeds with $\mathrm{T} 2^{*}$-weighted imaging at $1.5 \mathrm{~T}$ with dual-echo $\mathrm{T} 2^{\star}$-weighted imaging at $7 \mathrm{~T}$ and assess the reliability of the detection of microbleeds with the 2 field strengths.

\section{Materials and Methods}

\section{Patients}

Patients from the SMART study, ${ }^{26}$ without contraindications for $7 \mathrm{~T}$ MR imaging, were consecutively included between July 2008 and December 2009. The objectives of the SMART study are to determine the prevalence of vascular risk factors and concomitant arterial disease and to study the incidence of future cardiovascular events and its predictors in patients newly referred to our hospital with atherosclerotic disease. The SMART study and the $7 \mathrm{~T}$ imaging were approved by the medical ethics committee. Written informed consent was given by all patients. In total, 38 patients could be included in our study. The scans of 3 patients were used for a training session of the 2 observers and therefore were excluded from the final analysis. In one patient, $>20$ microbleeds were seen on the 7T scan. This was considerably more than in all other patients; the sum of all microbleeds in the remaining 34 patients on the $7 \mathrm{~T}$ scan was 36 . To prevent the results depending highly on the scan of this single patient with many microbleeds, this patient was considered as an outlier and excluded from the analysis. Therefore, the analysis was done on 34 patients.

\section{MR Imaging}

MR imaging was performed on a 1.5T whole-body system (Gyroscan ACS-NT; Philips Medical Systems, Best, the Netherlands) and a 7T whole-body system (Achieva; Philips Healthcare, Cleveland, Ohio). In all patients, a $3 \mathrm{D} \mathrm{T} 2^{\star}$-weighted imaging scan at $1.5 \mathrm{~T}$ and a $3 \mathrm{D}$ dual-echo $\mathrm{T} 2{ }^{\star}$-weighted MR imaging scan at $7 \mathrm{~T}$ were performed on the same day (MR imaging parameters shown in Table 1).

Because the dual-echo sequence at $7 \mathrm{~T}$ was originally designed as combination of an arteriogram and a venogram, the TE1 was used to make an inflow arteriogram. To control for intravoxel dephasing of flowing blood, the TE1 had to be short, with full flow compensation and partial Fourier encoding. The fat had to be suppressed; therefore, water and fat should be out of phase. For 7T, this is possible at $0.5,1.5$, $2.5 \mathrm{~ms}$, and so on. The minimal TE depends on the resolution and the gradient power, and in our case $2.5 \mathrm{~ms}$ is the optimum. Therefore, the TE1 was chosen at $2.5 \mathrm{~ms}$.

\begin{tabular}{lcc}
\hline \multicolumn{3}{l}{ Table 1: MR sequence parameters } \\
\hline Sequence Parameter & $1.5 \mathrm{~T}$ & $7 \mathrm{~T}$ \\
\hline TR (ms) & 25 & 20.0 \\
TE $(\mathrm{ms})$ & 35 & $2.5 / 15.0$ \\
Flip angle $\left({ }^{\circ}\right)$ & 10 & $16-24$ (variation \\
& & over slab) \\
Bandwidth (Hz/pixel) & 100 & 203 \\
Matrix size & $276 \times 226$ & $508 \times 399$ \\
Parallel imaging & Yes (acceleration & Yes (acceleration \\
& factor $=1.8)$ & factor $=2.5)$ \\
Flow compensation & No & Yes \\
Section thickness $(\mathrm{mm})$ & 0.8 & 0.6 \\
Gap (mm) & None & None \\
No. of sections & 125 & 167 \\
Acquired voxel size $(\mathrm{mm})$ & $0.8 \times 0.8 \times 0.8$ & $0.35 \times 0.4 \times 0.6$ \\
Interpolated voxel size $(\mathrm{mm})$ & $0.43 \times 0.43 \times 0.4$ & $0.35 \times 0.35 \times 0.3$ \\
Acquisition time & 6 min $56 \mathrm{~s}$ & 8 min $50 \mathrm{~s}$ \\
\hline
\end{tabular}

The TE2 was designed to make a venogram. Therefore, it was important to have a full echo and a flyback gradient, so the second readout gradient had the same polarity as the first readout gradient. ${ }^{27}$ To obtain enough sensitivity, the TE had to be relatively long, preferably between 20 and $30 \mathrm{~ms}$. A longer TE was not useful because the SNR would become too low. For the choice of the TE2, it is important that the TR is short enough to obtain good background suppression on the TE1 image (arteriogram). The flip angle was optimized for angiography in the TE1 and for venography in the TE2. Excitation pulses consisted of tilt-optimized nonsaturated excitation pulses with nominal flip angle variation of $16-24^{\circ}$ in the feet-head direction. With this flip angle, the TR had to be approximately $20 \mathrm{~ms} .{ }^{28}$ Here, a balance had to be found between a long enough TE for the venogram and a short enough TR for good background suppression in the arteriogram. As a compromise, the TE2 was chosen at $15 \mathrm{~ms}$.

The parameters of the $\mathrm{T} 2{ }^{\star}$-weighted imaging at $1.5 \mathrm{~T}$ were chosen to be comparable to the $7 \mathrm{~T}$ scan within the possibilities of the 1.5T MR imaging. Therefore, a scan with a high spatial resolution was made, with an acquired voxel size of $0.8 \times 0.8 \times 0.8 \mathrm{~mm}$. A PRESTO technique with a TE of $35 \mathrm{~ms}$ and a TR of $25 \mathrm{~ms}$ was used. During this sequence, additional field gradients shift the refocused gradient echo into the subsequent TR period, which results in a TE longer than the TR. In this sequence, the flip angle is not optimized for maximum SNR with the given TR, but small flip angles are needed to attenuate the contribution of spin echoes and stimulated echoes. ${ }^{29}$ With this technique, it is possible to obtain a longer TE with a relatively short $\mathrm{TR}$, resulting in a shorter scan time. This makes it possible to obtain a high-resolution scan with a long TE within a reasonable scan time. The use of PRESTO has been shown to result in a higher contrast-tonoise ratio compared with 3D-GRE for the visualization of microbleeds. $^{30}$

\section{Postprocessing}

The postprocessing of the data was performed on the console of the MR imaging system. The MIPs were reconstructed for transversal slabs (thickness, $3 \mathrm{~mm}$; 2-mm overlap; 100 sections) for the $1.5 \mathrm{~T}$ scan and for the TE1 and the TE2 of the 7T scan (Fig 1).

\section{Rating of Microbleeds}

All 1.5T and 7T scans were independently scored by 2 observers, both experienced neuroradiologists. The observers were trained in one session where they together evaluated scans of 3 patients with microbleeds. These 3 patients were excluded from the statistical analysis. 

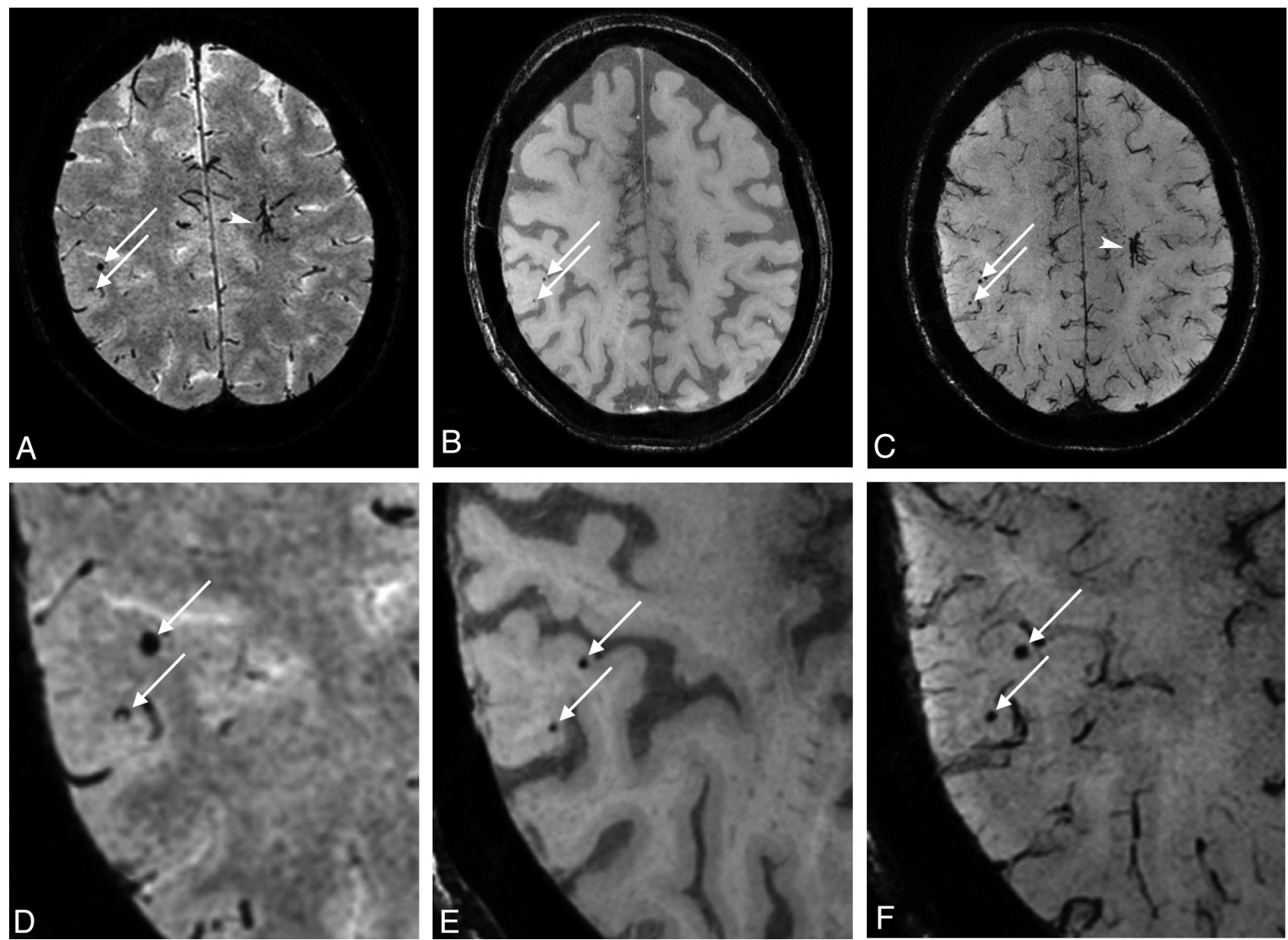

Fig 1. A, MIP of $3 \mathrm{~mm}$ thickness of the $1.5 \mathrm{~T}$ scan. Two microbleeds are visible (arrows).B, MIP of $3 \mathrm{~mm}$ thickness of the TE1 at 7T and of the TE2 image $(C)$. The $2 \mathrm{microbleeds}$ are also visible on both the TE1 and TE2 images (arrows). Note the developmental venous anomaly visible on the 1.5T scan (arrowhead) and very clearly demarcated on the TE2 of the 7T scan. $D-F$, Magnifications of the microbleeds on the 1.5T scan, the TE1 of the 7T scan, and the TE2 of the 7T scan, respectively.

For the 7T scans, TE1 and TE2 images were both used to score the microbleeds. The observers were blinded to the scan on the other field strength and to all clinical information. The scans of 10 patients were scored twice by one of the observers with a gap of 1 week to assess intraobserver reliability. The scans that were scored differently by the 2 observers were evaluated in a consensus meeting to obtain a final score.

The $1.5 \mathrm{~T}$ and $7 \mathrm{~T}$ scans were evaluated in a random order. There was a minimum gap of 1 week between the evaluation of a 1.5T and 7T scan of the same patient. The rating of the microbleeds was based on the Microbleed Anatomical Rating Scale (MARS). ${ }^{31}$ In this rating scale, microbleeds are scored as "definite" or "possible" microbleeds. In our study, we focused on definite microbleeds that were scored when they were visible on the TE1 or on the TE2 or on both echo images at 7T. Definite microbleeds are defined as small, rounded or circular, well-defined hypointense lesions within brain parenchyma with clear margins ranging from 2 to $10 \mathrm{~mm}$ on GRE T2*-weighted images in the MARS. In this study, we slightly adjusted this definition by excluding the size criterion. Due to the blooming effect, microbleeds appear larger on GRE T2*-weighted scans than their actual size. The blooming effect depends among other things on the field strength and the TE and will therefore be different for the 1.5T and 7T scan, which will result in different sizes for the same microbleeds at the 2 field strengths. This makes the size criterion not appropriate for this comparison study. Symmetrical areas of calcification in the basal ganglia, choroid plexus, and pineal gland were excluded, as were sig- nal intensity voids caused by sulcal vessels and low-signal intensity lesions thought to be signal intensity voids due to adjacent bone. ${ }^{3,10,31}$ Afterward, dissimilarities between the $1.5 \mathrm{~T}$ and the $7 \mathrm{~T}$ scans were assessed in a side-by-side comparison.

\section{Statistical Analysis}

Inter- and intraobserver reliability for the presence and absence of microbleeds was tested with Cohen $\kappa$ test. The $\kappa$-statistic was interpreted as poor $(0-0.20)$, fair $(0.21-0.40)$, moderate $(0.41-0.60)$, good $(0.61-0.80)$, or very good $(0.8-1) .{ }^{32}$ The reliability of the number of microbleeds was quantified by the ICC. The difference in prevalence of microbleeds between $1.5 \mathrm{~T}$ and $7 \mathrm{~T}$ scans was tested with the McNemar test. For the analysis of the difference in number of visible microbleeds between $1.5 \mathrm{~T}$ and $7 \mathrm{~T}$ scans, the Wilcoxon signed rank test was used. This analysis was done for all patients and for a selection of patients whose scans showed microbleeds at both field strengths. The analyses were performed by using the statistical software package SPSS (Version 15.0 for Windows; SPSS, Chicago, Illinois).

\section{Results}

Baseline characteristics of the 34 patients are shown in Table 2.

\section{Inter- and Intraobserver Reliability}

Table 3 shows the number of patients with and without definite microbleeds scored by both raters on $1.5 \mathrm{~T}$, resulting in a 


\begin{tabular}{|c|c|c|c|}
\hline & & & $\begin{array}{l}\text { All Patients } \\
(n=34)\end{array}$ \\
\hline \multicolumn{2}{|c|}{ Age $(y r)^{a}$} & \multicolumn{2}{|r|}{$58 \pm 12$} \\
\hline \multirow{2}{*}{\multicolumn{2}{|c|}{$\begin{array}{l}\text { Male gender (\%) } \\
\text { Inclusion in SMART study with }\end{array}$}} & \multicolumn{2}{|r|}{85} \\
\hline & \\
\hline Vascular risk factors & & \multicolumn{2}{|r|}{50} \\
\hline Cerebrovascular disea & & \multicolumn{2}{|r|}{26} \\
\hline Peripheral artery dise & & \multicolumn{2}{|r|}{15} \\
\hline Cardiovascular diseas & & \multicolumn{2}{|r|}{9} \\
\hline \multicolumn{4}{|c|}{ 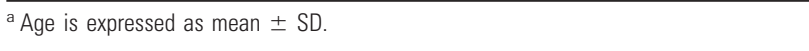 } \\
\hline \multicolumn{4}{|c|}{$\begin{array}{l}\text { Table 3: Number of patients with visible microbleeds at } 1.5 \mathrm{~T} \text { for the } \\
2 \text { observers }\end{array}$} \\
\hline & \multicolumn{2}{|c|}{ Observer 2} & \\
\hline & Microbleeds & Microbleeds & \\
\hline $1.5 \mathrm{~T}$ & Absent & Present & Total \\
\hline \multicolumn{4}{|l|}{ Observer 1} \\
\hline Microbleeds absent & 21 & 6 & 27 \\
\hline Microbleeds present & 3 & 4 & 7 \\
\hline Total & 24 & 10 & 34 \\
\hline
\end{tabular}

Table 4: Number of patients with visible microbleeds at $7 T$ for the 2 observers

\begin{tabular}{lccr}
\hline & \multicolumn{3}{c}{ Observer 2 } \\
\cline { 2 - 3 } & $\begin{array}{c}\text { Microbleeds } \\
\text { Absent }\end{array}$ & $\begin{array}{c}\text { Microbleeds } \\
\text { Present }\end{array}$ & Total \\
\hline Observer 1 & & & \\
Microbleeds absent & 18 & 7 & 25 \\
$\quad$ Microbleeds present & 2 & 7 & 9 \\
Total & 20 & 14 & 34 \\
\hline
\end{tabular}

\begin{tabular}{|c|c|c|c|}
\hline \multirow[b]{2}{*}{ Consensus 2 observers } & \multicolumn{2}{|c|}{ 7T } & \multirow[b]{2}{*}{ Tota } \\
\hline & $\begin{array}{c}\text { Microbleeds } \\
\text { Absent }\end{array}$ & $\begin{array}{c}\text { Microbleeds } \\
\text { Present }\end{array}$ & \\
\hline \multicolumn{4}{|l|}{$1.5 \mathrm{~T}$} \\
\hline Microbleeds absent & 17 & 9 & 26 \\
\hline Microbleeds present & 0 & 8 & 8 \\
\hline Total & 17 & 17 & 34 \\
\hline
\end{tabular}

fair interobserver reliability $(\kappa=0.30)$. At 7T, the interobserver reliability for definite microbleeds was moderate $(\kappa=$ 0.42 ) (Table 4 ). The intraobserver reliability was moderate at $1.5 \mathrm{~T}(\kappa=0.52)$ and good at $7 \mathrm{~T}(\kappa=0.80)$. For the number of microbleeds, moderate interobserver reliability was demonstrated for the $1.5 \mathrm{~T}(\mathrm{ICC}=0.50)$ and good reliability for the $7 \mathrm{~T}(\mathrm{ICC}=0.61)$ scans. The intraobserver reliability for the number of microbleeds was moderate at $1.5 \mathrm{~T}(\mathrm{ICC}=0.59)$ and very good at $7 \mathrm{~T}(\mathrm{ICC}=0.94)$.

\section{Presence and Number of Microbleeds}

Table 5 shows the number of patients with and without microbleeds at $1.5 \mathrm{~T}$ and $7 \mathrm{~T}$ after consensus. One or more microbleeds were found on $1.5 \mathrm{~T}$ scans in $8(23.5 \%)$ patients and on $7 \mathrm{~T}$ scans in $17(50 \%)$ patients. The number of patients with microbleeds at $7 \mathrm{~T}$ was significantly higher than at $1.5 \mathrm{~T}(P=$ .002). There were no patients in whom microbleeds were scored at $1.5 \mathrm{~T}$ that were not scored at $7 \mathrm{~T}$.
In the 8 patients with microbleeds on the $1.5 \mathrm{~T}$ scans, 15 microbleeds in total were scored (median number of microbleeds, 0.0 ; range, $0-6$ ). Of these 15 microbleeds, 7 were located frontal, 3 parietal, 2 occipital, 1 temporal, 1 in the basal ganglia, and 1 in the deep and periventricular white matter. In the 17 patients with microbleeds on the 7T scans, 36 microbleeds in total were scored (median, 0.5 ; range, $0-5$ ), a number that was significantly more than at $1.5 \mathrm{~T}(P=.003)$. Of the 36 microbleeds scored on the 7T, 14 were located frontal, 7 parietal, 5 occipital, 5 temporal, 2 in the basal ganglia, 2 in the cerebellum, and 1 in the thalamus. In all 8 patients with microbleeds on $1.5 \mathrm{~T}$, microbleeds also were present on the $7 \mathrm{~T}$ scan. The median number of microbleeds in these patients with visible microbleeds at both field strengths was 1.0 (range, $1-6)$ at $1.5 \mathrm{~T}$ and 2.0 (range, $1-5)$ at $7 \mathrm{~T}$. However, this difference was not significant $(P=.236)$.

\section{Side-by-Side Comparison}

Twenty-seven microbleeds (75\%) out of the 36 microbleeds that were scored on $7 \mathrm{~T}$ scans were not scored on $1.5 \mathrm{~T}$ scans. Side-by-side comparison of $1.5 \mathrm{~T}$ and $7 \mathrm{~T}$ scans showed that 13 $(48 \%)$ of the microbleeds that were scored at $7 \mathrm{~T}$ and not on $1.5 \mathrm{~T}$ were small microbleeds on the $7 \mathrm{~T}$ scans, which were not visible on the $1.5 \mathrm{~T}$ scan. Two of these microbleeds were not visible due to overlap of a venous structure. Five other microbleeds $(19 \%)$ were visible at $1.5 \mathrm{~T}$, however they were located in the vicinity of a venous structure and therefore could not be reliably distinguished as a microbleed. Four microbleeds (15\%) were visible as hypointense lesions, but not as a typical well-defined, round-shaped lesion, as illustrated in Fig 2. As shown in Fig 3, 3 microbleeds (11\%) that were not scored on the $1.5 \mathrm{~T}$ scans were in retrospect visible as very small lesions that could hardly be distinguished from the background noise. One microbleed (4\%) was missed, which was located near the hypointense basal ganglia; on the 7T scan, this microbleed was only visible on the TE1 image. One other microbleed (4\%) was just overlooked by both observers.

Of the total of 15 microbleeds that were scored on 1.5T scans, 5 microbleeds (33\%) were not scored on 7T scans. In retrospect, these microbleeds were visible as very small lesions on the 7T scans and were missed by the observers, or they were defined as no definite microbleed during the consensus meeting. An example of one of those microbleeds is shown in Fig 4.

\section{Discussion}

In this study, we found a fair-to-moderate inter- and intraobserver reliability for the presence of microbleeds by using 3D $\mathrm{T} 2{ }^{*}$-weighted imaging at $1.5 \mathrm{~T}$ and moderate-to-good reliability by using dual-echo $\mathrm{T} 2^{\star}$-weighted imaging at $7 \mathrm{~T}$. For the number of microbleeds, the reliability was better; it was moderate at $1.5 \mathrm{~T}$ and good to very good at $7 \mathrm{~T}$. Overall, we found an increased reliability for both presence and number of microbleeds at $7 \mathrm{~T}$ compared with 1.5T. Cerebral microbleeds were detected in more patients at $7 \mathrm{~T}$ than at $1.5 \mathrm{~T}$. Furthermore, the number of microbleeds detected was higher at $7 \mathrm{~T}$.

Besides the detection of microbleeds in more patients and a higher number of microbleeds at 7T, the reliability of the detection also improves. Compared with 1.5T, both inter- and intraobserver reliability improved at $7 \mathrm{~T}$. This might be due to the higher resolution, increased SNR, and the use of the dual- 

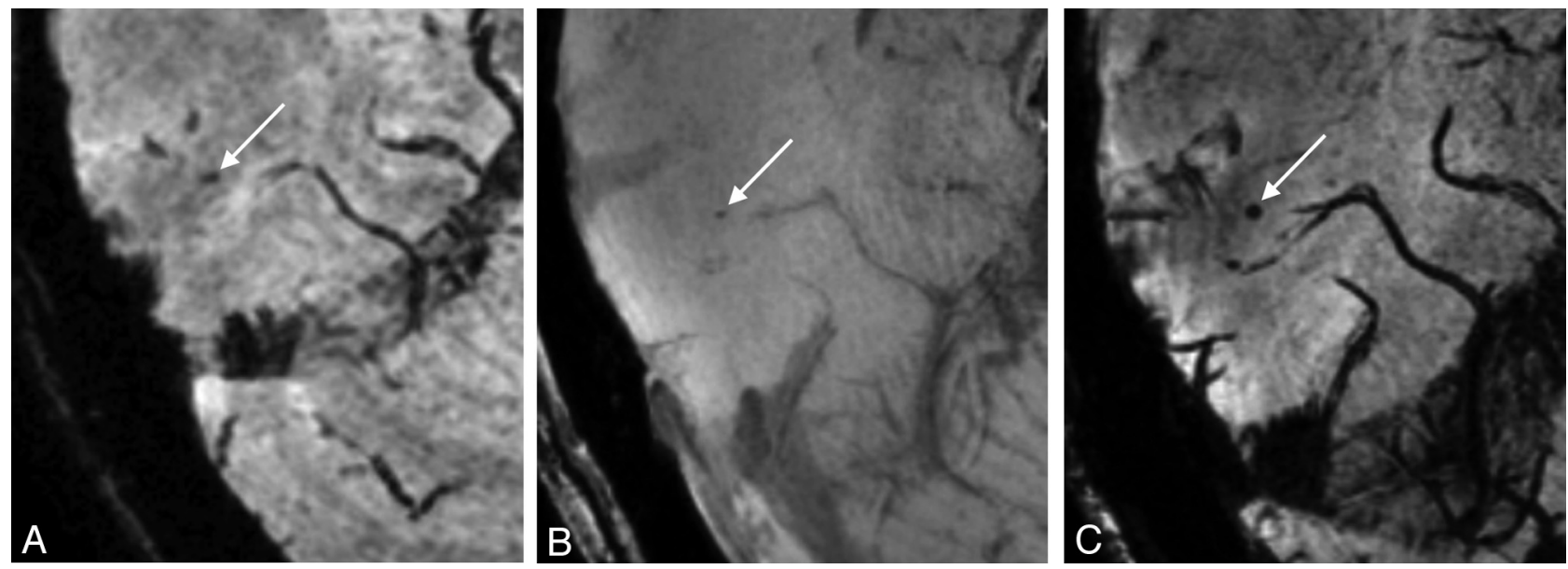

Fig 2. A, On this 1.5T scan, a hypointense lesion (arrow) is visible that is not a typical well-defined, round-shaped lesion as in the definition of a microbleed. This lesion was not scored as a microbleed on the 1.5T scan. In $B$ and $C$, this same lesion is visible on the $7 T$ scan. On the TE1 image $(B)$, it is visible as a faint, round hypointensity and on the TE2 image $(C)$, it is a clearly visible round hypointensity (arrow). On the 7T scan, both raters scored this lesion as a microbleed.
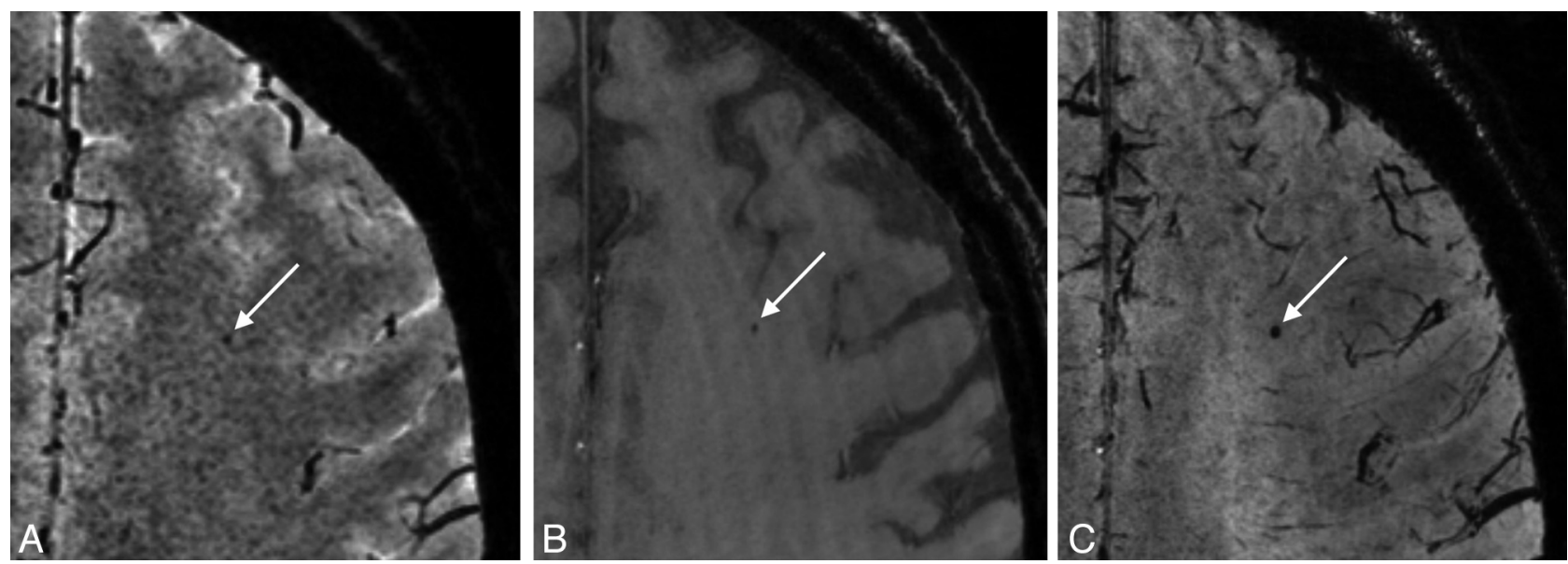

Fig 3. $A, 1.5 \mathrm{~T}$ scan shows a hypointense lesion (arrow) that can hardly be distinguished from noise and was not scored as a microbleed. On both the TE1 image $(B)$ and TE2 image ( $C)$ of the 7T scan, this hypointense lesion is visible as a typical microbleed, showing enlargement at the TE2 due to the blooming effect. This lesion was scored as a microbleed by both observers.
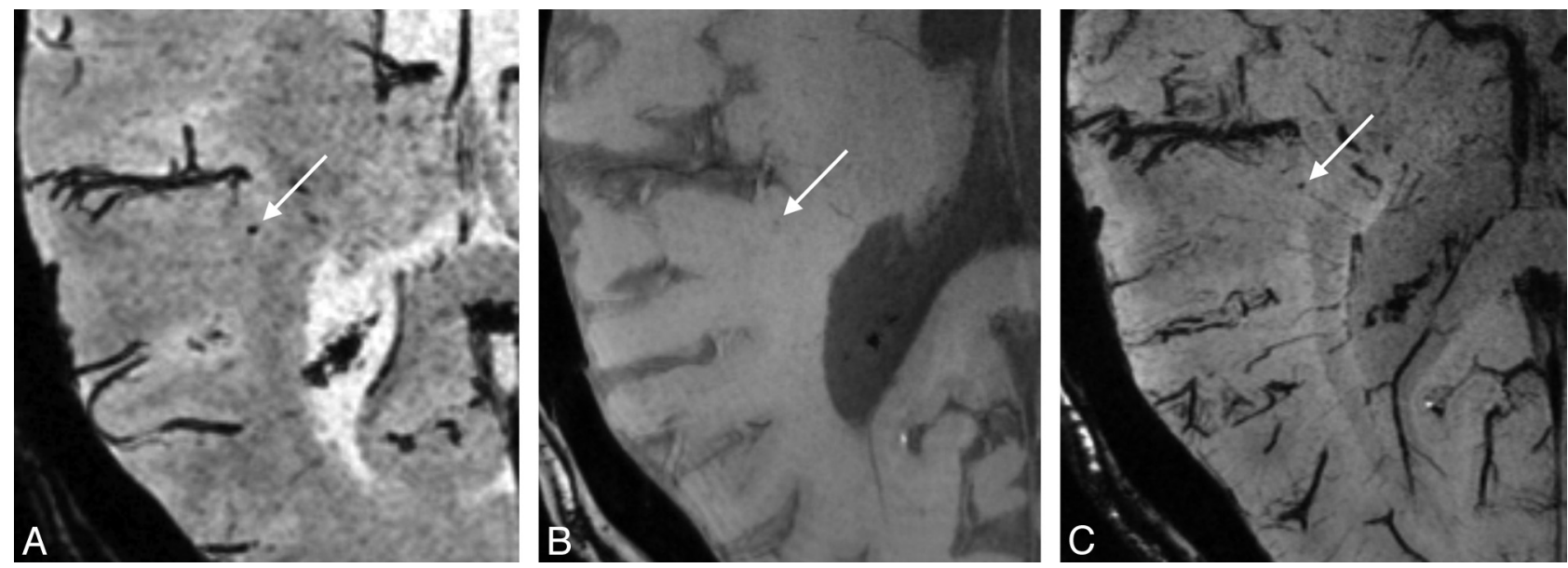

Fig 4. $A$, 1.5T scan shows a well-defined, round, hypointense lesion (arrow) that was scored as a microbleed. However, on the TE1 of the 7T scan (B), this lesion is only visible as a very faint hypointensity and on the TE2 image $(C)$ it appears as a small microbleed. On the 7T scan, this lesion was not scored as a microbleed.

echo sequence at 7T. Recently, we showed that the TE1 image provides a good contrast of the dark microbleeds against a homogeneous, more hyperintense signal intensity of the brain tissue and that $85 \%$ of all microbleeds are visible on the TE1 image. ${ }^{25}$ If a microbleed is visible on the TE1 image and also shows a blooming effect on the TE2, it is more easily classified 
as a definite microbleed, whereas the $1.5 \mathrm{~T}$ scan provides only one single-echo image of the microbleed. In addition, the contrast between microbleeds and the background is less on the $1.5 \mathrm{~T}$ scan and the resolution is slightly lower, making it more difficult to distinguish microbleeds from the background noise and from other hypointense structures compared with the 7T scan. The use of the TE1 image at 7T is helpful to distinguish microbleeds from deoxyhemoglobin in veins and ferritin in the basal ganglia, whereas microbleeds can be missed by overlap of veins or the hypointense appearance of ferritin-containing basal ganglia on the $1.5 \mathrm{~T}$ scan or on the TE2 of the 7 T scan. ${ }^{25}$

Previous studies comparing the detection of microbleeds at $3 \mathrm{~T}$ and $1.5 \mathrm{~T}$ found significantly more microbleeds at $3 \mathrm{~T}$. In addition, the microbleed conspicuity improved at $3 \mathrm{~T} .{ }^{18,23}$ These results are in line with our results of a better detection of microbleeds at 7T, indicating that the detection of microbleeds improves with increasing field strength. The higher resolution, higher SNR, and increased susceptibility effect at 7T increased the conspicuity of smaller microbleeds. This resulted in the detection of more and smaller microbleeds. However, a drawback is that very small microbleeds were hard to distinguish from background noise, and small microbleeds were easily missed by the observers.

Unexpectedly, some microbleeds were scored at $1.5 \mathrm{~T}$ but not at 7T. A possible reason for this is that at 7T more and smaller veins are visible, making it harder to distinguish small microbleeds from small veins. This is illustrated by Fig 4, where the microbleed that was missed on the 7T scan was located near a very small vein and could therefore be mistaken as part of the vein.

Nowadays, SWI is increasingly used for the detection of microbleeds. ${ }^{18,33}$ In SWI, the magnitude and phase images of the $\mathrm{T} 2{ }^{*}$-weighted scan are combined to create enhanced contrast between tissues with different susceptibilities. ${ }^{34}$ However, SWI depends strongly on the voxel aspect ratio, with an optimal voxel aspect ratio of $1: 1: 4$; we used more isotropic voxels (isotropic voxels at $1.5 \mathrm{~T}$ and an aspect ratio of 1:1.3:1.7 at 7T). ${ }^{35}$ The additional value of SWI will be minimal for scans with (near) isotropic voxels, and we therefore chose $\mathrm{T}^{*}$ weighted imaging instead of SWI for the detection of microbleeds in our study.

A limitation of the study is the relatively low observer reliability at $1.5 \mathrm{~T}$. The study by Gregoire et $\mathrm{al}^{31}$ describing the MARS shows a very good observer reliability by using MARS at $1.5 \mathrm{~T}$. There are several possible explanations for this difference. First, Gregoire et $\mathrm{al}^{31}$ excluded patients with one definite microbleed from the analysis, which substantially improved their interobserver reliability; we did not exclude patients with only one microbleed. Besides that, in the study of Gregoire et $\mathrm{al}^{31}$ a section thickness of $5 \mathrm{~mm}$ with a gap of $1.5 \mathrm{~mm}$ was used, which is a large difference from our $0.8-\mathrm{mm}$ sections. The use of thin sections results in many more sections and also more small microbleeds will become visible. Those small microbleeds are easily missed by the observers, especially when they have to evaluate many sections, inducing differences between the scores of observers. Furthermore, Gregoire et $\mathrm{al}^{31}$ did not score microbleeds $<2 \mathrm{~mm}$, whereas we did not use a minimum diameter in our study. In other studies at $1.5 \mathrm{~T}$, observer reliabilities vary from fair to very good; $;^{3,7,36}$ probably this is also due to differences in scan parameters and definition of microbleeds.

Another limitation of this study is that the scan parameters of the $1.5 \mathrm{~T} \mathrm{~T} 2{ }^{\star}$-weighted sequence were different from the 7T dual-echo sequence. This makes it difficult to evaluate the net effect of the increased field strength. However, when going to higher field strength, it is not feasible to maintain the same scan parameters. Therefore, we tried to optimize both scans as much as possible for the corresponding field strength within a reasonable scan time. The TE is the parameter that most affects sensitivity in $\mathrm{T} 2{ }^{\star}$-weighted MR imaging. ${ }^{10} \mathrm{The} \mathrm{TE}$ at $1.5 \mathrm{~T}$ is 35 $\mathrm{ms}$, whereas it is only $15 \mathrm{~ms}$ for the TE2 image at 7T. A longer TE gives more time for dephasing, resulting in an increase in diameter of microbleeds and also an increase in the number of microbleeds detected. ${ }^{19}$ However, within the dual-echo sequence at 7T, a balance had to be found between a TE, which gives enough dephasing and a short TR, which is necessary for good background suppression in the TE1 image. Therefore, the TE could not be equally long in the 7T sequence as in the $1.5 \mathrm{~T}$ sequence. The use of a longer TE at $7 \mathrm{~T}$ could result in the detection of more microbleeds. However, because other structures with a high susceptibility, such as veins, or iron deposition in the basal ganglia, will also become larger, this may lead to more overlap of microbleeds by these brain structures. Therefore, we think it is important to use 2 TEs to combine the benefits of a short and a long TE.

Although interest in cerebral microbleeds has greatly increased, their relevance in clinical practice remains unclear. ${ }^{37}$ It is possible that the current studies, which are now mainly done at $1.5 \mathrm{~T}$, only detect the tip of the iceberg of all microbleeds present in the brain. Scanning at higher field strengths, such as 7T, might be necessary to improve the detection of microbleeds and the reliability of this detection. Because microbleeds can be detected in more patients and also the lesion load can be determined more accurately, this could result in more detailed and consistent information about the clinical significance of microbleeds. Furthermore, microbleeds are regarded as markers of vascular pathology and it can be interesting to study changes in patients with cerebral amyloid angiopathy, hypertensive vasculopathy, or Alzheimer disease. ${ }^{10}$ The improved detection of microbleeds at 7T may be useful for follow-up studies with small changes in microbleed load over time. Furthermore, good detection methods for microbleeds are needed for future studies to determine whether microbleeds should affect clinical decision making, to identify their relation with other signs of cerebral small vessel disease, and to describe their independent contribution to cognitive and neurologic dysfunction. ${ }^{10}$

\section{Conclusions}

We found that presence and number of detected microbleeds are higher and also that the reliability of the detection of microbleeds improves with a $3 \mathrm{D}$ dual-echo $\mathrm{T} 2^{\star}$-weighted sequence at $7 \mathrm{~T}$ compared with a $3 \mathrm{D} \mathrm{T} 2^{*}$-weighted sequence at $1.5 \mathrm{~T}$.

\section{References}

1. Cordonnier C, van der Flier WM, Sluimer JD, et al. Prevalence and severity of microbleeds in a memory clinic setting. Neurology 2006;66:1356-60

2. Dichgans M, Holtmannspötter M, Herzog J, et al. Cerebral microbleeds in 
CADASIL: a gradient-echo magnetic resonance imaging and autopsy study. Stroke 2002;33:67-71

3. Jeerakathil T, Wolf PA, Beiser A, et al. Cerebral microbleeds: prevalence and associations with cardiovascular risk factors in the Framingham Study. Stroke 2004;35:1831-35

4. Kikuta K, Takagi Y, Nozaki K, et al. Asymptomatic microbleeds in Moyamoya disease: $\mathrm{T} 2{ }^{\star}$-weighted gradient-echo magnetic resonance imaging study. J Neurosurg 2005;102:470-75

5. Koennecke HC. Cerebral microbleeds on MRI: prevalence, associations, and potential clinical implications. Neurology 2006;66:165-71

6. Mori N, Miki Y, Kikuta K, et al. Microbleeds in Moyamoya disease: susceptibility-weighted imaging versus $\mathrm{T} 2{ }^{*}$-weighted imaging at 3 Tesla. Invest Radiol 2008;43:574-79

7. Vernooij MW, van der Lugt A, Ikram MA, et al. Prevalence and risk factors of cerebral microbleeds: the Rotterdam Scan Study. Neurology 2008;70:1208-14

8. Wardlaw JM, Lewis SC, Keir SL, et al. Cerebral microbleeds are associated with lacunar stroke defined clinically and radiologically, independently of white matter lesions. Stroke 2006;37:2633-36

9. Fazekas F, Kleinert R, Roob G, et al. Histopathologic analysis of foci of signal loss on gradient-echo $\mathrm{T} 2{ }^{\star}$-weighted MR images in patients with spontaneous intracerebral hemorrhage: evidence of microangiopathy-related microbleeds. AJNR Am J Neuroradiol 1999;20:637-42

10. Greenberg SM, Vernooij MW, Cordonnier C, et al. Cerebral microbleeds: a guide to detection and interpretation. Lancet Neurol 2009;8:165-74

11. Fiehler J, Albers GW, Boulanger JM, et al. Bleeding Risk Analysis in Stroke Imaging before thromboLysis (BRASIL): pooled analysis of $\mathrm{T} 2{ }^{*}$-weighted magnetic resonance imaging data from 570 patients. Stroke 2007;38:2738-44

12. Gregoire SM, Jäger HR, Yousry TA, et al. Brain microbleeds as a potential risk factor for antiplatelet-related intracerebral haemorrhage: hospital-based, case-control study. J Neurol Neurosurg Psychiatry 2010;81:679-84

13. Soo YO, Yang SR, Lam WW, et al. Risk vs benefit of anti-thrombotic therapy in ischaemic stroke patients with cerebral microbleeds. I Neurol 2008;255: $1679-86$

14. Vernooij MW, Van der Lugt A, Breteler MM. Risk of thrombolysis-related hemorrhage associated with microbleed presence. Stroke 2008;39:e115

15. Vernooij MW, Heeringa J, de Jong GJ, et al. Cerebral microbleed preceding symptomatic intracerebral hemorrhage in a stroke-free person. Neurology 2009;72:763-65

16. Henneman WJ, Sluimer JD, Cordonnier C, et al. MRI biomarkers of vascular damage and atrophy predicting mortality in a memory clinic population. Stroke 2009;40:492-8

17. Werring DJ, Frazer DW, Coward LJ, et al. Cognitive dysfunction in patients with cerebral microbleeds on T2*-weighted gradient-echo MRI. Brain 2004; 127(pt 10):2265-75

18. Nandigam RN, Viswanathan A, Delgado P, et al. MR imaging detection of cerebral microbleeds: effect of susceptibility-weighted imaging, section thickness, and field strength. AJNR Am J Neuroradiol 2009;30:338-43

19. Vernooij MW, Ikram MA, Wielopolski PA, et al. Cerebral microbleeds: accel- erated 3D $\mathrm{T}^{*}$-weighted GRE MR imaging versus conventional 2D T2* weighted GRE MR imaging for detection. Radiology 2008;248:272-77

20. Roob G, Schmidt R, Kapeller P, et al. MRI evidence of past cerebral microbleeds in a healthy elderly population. Neurology 1999;52:991-94

21. Tatsumi S, Ayaki T, Shinohara M, et al. Type of gradient recalled-echo sequence results in size and number change of cerebral microbleeds. AJNR Am J Neuroradiol 2008;29:e13

22. Reichenbach JR, Venkatesan R, Yablonskiy DA, et al. Theory and application of static field inhomogeneity effects in gradient-echo imaging. J Magn Reson Imaging 1997;7:266-79

23. Stehling C, Wersching $\mathrm{H}$, Kloska SP, et al. Detection of asymptomatic cerebral microbleeds: a comparative study at 1.5 and 3.0 T. Acad Radio 2008;15:895-900

24. Haacke EM, Mittal S, Wu Z, et al. Susceptibility-weighted imaging: technical aspects and clinical applications, part 1. AJNR Am J Neuroradiol 2009;30:19-30

25. Conijn MM, Geerlings MI, Luijten PR, et al. Visualization of cerebral microbleeds with dual-echo $\mathrm{T} 2{ }^{\star}$-weighted magnetic resonance imaging at 7.0 T. J Magn Reson Imaging 2010;32:52-59

26. Simons PC, Algra A, van de Laak MF, et al. Second Manifestations of ARTeria disease (SMART) study: rationale and design. Eur J Epidemiol 1999;15:773-81

27. Du YP and Jin Z. Simultaneous acquisition of MR angiography and venography (MRAV). Magn Reson Med. 2008;59:954-58

28. Hendrikse J, Zwanenburg JJ, Visser F, et al. Noninvasive depiction of the lenticulostriate arteries with time-of-flight MR angiography at 7.0 T. Cerebrovasc Dis 2008;26:624-29

29. Liu G, Sobering G, Duyn J, et al. A functional MRI technique combining principles of echo-shifting with a train of observations (PRESTO). Magn Reson Med 1993;30:764-68

30. Tsuboyama T, Imaoka I, Shimono T, et al. T2*-sensitized high-resolution magnetic resonance venography using 3D-PRESTO technique. Magn Reson Med Sci 2008;7:73-77

31. Gregoire SM, Chaudhary UJ, Brown MM, et al. The Microbleed Anatomical Rating Scale (MARS): reliability of a tool to map brain microbleeds. Neurology 2009;73:1759-66

32. Landis JR and Koch GG. The measurement of observer agreement for categorical data. Biometrics 1977;33:159-74

33. Kirsch W, McAuley G, Holshouser B, et al. Serial susceptibility weighted MR measures brain iron and microbleeds in dementia. J Alzheimers Dis 2009 May

34. Bradley WG Jr. MR appearance of hemorrhage in the brain. Radiology 1993;189:15-26

35. Xu Y and Haacke EM. The role of voxel aspect ratio in determining apparent vascular phase behavior in susceptibility weighted imaging. Magn Reson Imaging 2006;24:155-60

36. Cordonnier C, Potter GM, Jackson CA, et al. Improving interrater agreement about brain microbleeds: development of the Brain Observer MicroBleed Scale (BOMBS). Stroke 2009;40:94-99

37. Cordonnier C. Brain microbleeds. Pract Neurol 2010;10:94-100 